little is known about patterns of anxiety across this period. This study aimed to 1) assess patterns of anxiety and depression across pregnancy and the postpartum, 2) investigate associations between antenatal mood and HPA axis hormones and 3) determine the extent to which antenatal anxiety, depression and HPA axis activity predict postnatal mood disorders.

Methods: Participants were recruited antenatally as part of a prospective study undertaken at the Royal Hospital for Women, Sydney. Ninety-four women completed self-report measures of anxiety and depression at 30-32 and 36-38 weeks gestation, and at 6 months postpartum. They were also administered a structured diagnostic interview (MINI-Plus) at 36-38 weeks gestation and at 6 months postpartum to determine the presence of DSM-IV anxiety and depression. Blood samples were collected at 30-32 weeks gestation for bioassays of HPA axis hormones (CRH, ACTH and cortisol).

Results: The data indicate significant stability in maternal mood across pregnancy and the postpartum and associations between anxiety and depression were moderate-high at each assessment. Despite the stability of depression, an anxiety disorder in pregnancy appears to be a greater risk factor for a postnatal anxiety [odds ratio $(\mathrm{OR})=10.20, P<0.005$ ] or depressive disorder $(\mathrm{OR}=7.90, P<0.005)$ than antenatal depression. Antenatal neuroendocrine parameters were unrelated to either antenatal or postnatal anxiety or depression.

Conclusion: These results clearly highlight the importance of anxiety in both the pre- and postnatal periods.

\section{Phenotypic correlates of the serotonin transporter gene}

\section{A Finch', K Baikie, ${ }^{1,2}$, P Mitchell2, G Parker ${ }^{1,2}$, J Reddy ${ }^{1,2}$, PR Schofield ${ }^{3}$, T Showyin ${ }^{1,2}$, J Siegel ${ }^{1,2}$, L Wedgwood ${ }^{1,2}, \mathrm{~K}$ Wilhelm ${ }^{2,4}$}

'The Black Dog Institute; ${ }^{2}$ School of Psychiatry, UNSW; ${ }^{3}$ Prince of Wales Medical Research Institute; and ${ }^{4}$ Consulation Liaison Psychiatry, St Vincent's Hospital, Sydney, Australia

Background: A genetic variation within the promoter region of the serotonin transporter (5-HTT) gene has been found to moderate the effect of stressful life events on onset of major depression (Caspi et al. 2003; Wilhelm et al. 2006). This paper examines for observable characteristics underlying the genetic liability to depression following stressful events associated with differing 5-HTT genotypes within two study samples. Method: Study 1 - 'diabetes study'. Commencing in July 2006, patients presenting to a hospital-based diabetes clinic were recruited. Participants provided data on psychiatric diagnosis, personality traits (NEO) and coping styles (COPE), as well as provided saliva samples for genetic analysis. Study 2 - 'teachers cohort study'. Between 1978 and 1998, episodes of major depression, life events, coping behaviours and trait anxiety measures (EPQ, TCI) were recorded at 5 yearly intervals. In 2003, blood or saliva samples were collected for genetic analysis.

Results: Associations between the 5-HTT gene and candidate phenotypes (trait anxiety and coping styles) were examined using preliminary data from the diabetes sample (anticipated $n>100$ ). For the teachers cohort study, no associations between the 5-HTT genotype and trait anxiety were found for those who provided genetic material $(n=128)$. There were, however, significant differences on the coping behaviours used by differing genotype groups when under stress.

Conclusions: These findings raise the possibility of a genetic disposition to emotional reactivity to stressors that may predispose individuals to use different coping strategies. Replication of these findings will be examined within the diabetes sample.

\section{Clozapine and cardiotoxicity: echocardiography findings from Barwon Health}

\section{J Fitzsimons' ${ }^{1}$, T Lambert', S Dodd ${ }^{2}$, C Pantelis', T Callaly'2, B Kuluris², M Berk'}

'The University of Melbourne; and 'Barwon Health, Melbourne, Australia

Background: Clozapine continues to have a unique efficacy profile that to date has not been matched or enhanced by other second-generation antipsychotics. Although agranulocytosis is a well-documented vulnerability for these patients, other serious risks, such as myocarditis and cardiomyopathy, are less well recognized and there remains a dearth of examination in this area. The current study aims to investigate changes in cardiac functioning in a group of patients treated for the first time with clozapine.

Methods: Transthoracic echocardiograms were conducted on 77 clozapine-naïve patients, prior to commencing clozapine treatment (time 1) and were repeated after 6-12 months (time 2). Patient psychiatric and medication history were documented, as were full white blood count, troponin 1 and creatinine kinase results. The rate of clozapine titration was also recorded.

Results: Preliminary analyses of the data set indicate a decrease in left ventricular shortening, a measure of ventricular contractility, from time 1 (pre clozapine) to time 2. Further analyses will be presented. 